\title{
MUSCULACIÓN EN GIMNASIOS: APROXIMACIÓN A LOS MOTIVOS PARA ENTRENAR CON PESAS
}

\author{
Félix Arbinaga Ibarzábal y José María García García \\ Avda. José Fariña 56-5ㄷ, Huelva 21006 (España) \\ E-mail: arbinaga@wanadoo.es
}

\begin{abstract}
Resumen
Arbinaga Ibarzábal, F. y García García, J. M. (2002). Musculación en gimnasios: aproximación a los motivos para entrenar con pesas. Revista de Ciencias del Ejercicio y la Salud, 2(2), 62-69. El estudio piloto que presentamos pretende llevar a cabo un primer análisis sobre la motivación que muestra un grupo de 55 personas $(72,73 \%$ de hombres y $27,27 \%$ de mujeres) para realizar actividades de musculación mediante entrenamiento con pesas en gimnasios. El instrumento utilizado para evaluar la motivación ha sido la Escala de Motivación en el Deporte (E.M.D) de Brière, Vallerand, Blais y Pelletier (1995) adaptada por López (2000). De los resultados se destaca que el motivo principal para iniciarse en el entrenamiento con pesas en gimnasios varía entre hombres y mujeres. Éstas, en el 66,7\% de los casos, dicen acudir para controlar el peso frente al $15 \%$ de los hombres; mientras éstos acuden principalmente para mantener la forma física (50\%). La muestra nos refleja una población joven, siendo las mujeres quienes suelen puntuar más en el factor de regulación introyectada $(p<0.05)$ mientras los varones lo hacen en regulación externa $(p<0.001)$. En este sentido, son los varones quienes más desean parecerse a compañeros con mayor desarrollado muscular $(p=0.01118)$, siendo aquellos que más se comparan los que desearían llegar a competir $(p=0.01967)$. Éstos últimos se ven en mayor medida influenciados por una regulación externa $(p=0.011)$ y una motivación intrínseca al conocimiento $(p=0.005)$. PALABRAS CLAVES: Motivación, entrenamiento, musculación, gimnasio.
\end{abstract}

\section{INTRODUCCIÓN}

Los profesionales de la psicología aplicada al deporte y la actividad física han considerado la motivación en la ejecución deportiva como unas de las preocupaciones prioritarias de trabajo; existiendo diversas investigaciones sobre los motivos que llevan a los jóvenes a practicar o abandonar deportes concretos (García Ferrando, 1991, Balaguer y Atienza, 1994, Rodríguez, 1998). Esto ha sido así, dado que cuando se logra el interés y la atención del grupo con el que se trabaja puede establecerse las condiciones óptimas para la consecución de los objetivos propuestos (Gordillo, 1992) existiendo la posibilidad de diseñar programas deportivos que cubran las necesidades de los atletas, tanto deportiva como en su desarrollo personal (Gould, 1982).

Aunque la motivación ha sido definida de muchas y variadas maneras, en general, la mayoría coincide en referirla a la intensidad y la dirección del comportamiento (Morilla, 1994); de forma genérica suele entenderse que la motivación es la causa de la conducta, el por qué del comportamiento. Actualmente se considera a la motivación como el resultado en la interacción de una amplia gama de factores personales y situacionales que actúan sobre el individuo. Por otra parte, han existido variados sistemas de clasificación de las motivaciones humanas; si bien, una posibilidad que favorece la operacionalización de los motivos es considerarla al menos en tres posibles dimensiones: motivación extrínseca, motivación intrínseca y amotivación (Deci, 1971; Deci y Ryan, 1985). Y a su vez, en cada una de las dimensiones, ha sido posible discriminar diversos factores recogidos en escalas específicas (Bière, Vallerand, Blais, y Pelletier, 1995).

La Motivación Intrínseca es definida como el hecho de practicar una actividad por ella misma o por el placer generado en su práctica (Vallerand y Halliwell, 1983). Se han distinguido tres tipos de motivación intrínseca relacionadas con el conocimiento, el cumplimiento y la estimulación. 
La Motivación Interna relacionada con el Conocimiento se define por el placer y la satisfacción que uno experimenta mientras aprende algo nuevo; relacionándose constructos como exploración, curiosidad, objetivos de aprendizaje etc. La Motivación Interna relacionada con el Cumplimiento es entendida cuando el sujeto se centra más en los procesos de logro que sobre los resultados. Por su parte, la Motivación Interna relacionada con la Estimulación, opera cuando alguien realiza una acción a fin de experimentar sensaciones.

En el caso de la Motivación Extrínseca ésta se basa en tres conceptos principales: recompensa, castigo e incentivo. Se ha distinguido entre la motivación extrínseca referida a regulación externa, referida a una regulación introyectada y a la identificación.

En la Motivación Extrínseca referida a la Regulación Externa, la conducta es regulada a través de medios externos tales como premios y castigos. En el caso de la Motivación Extrínseca referida a la Regulación Introyectada, el sujeto internaliza las razones para sus acciones, pero esta internalización está limitada a las contingencias externas. Por último, la Motivación Extrínseca referida a la Identificación se basa en que el sujeto juzga su conducta como importante para él y la percibe como escogida por él mismo; entonces la internalización de los motivos externos se regula a través de la identificación.

Por último, la Amotivación corresponde a un estado de no regulación y sería diferente de las motivaciones intrínsecas o extrínsecas. (Deci y Ryan, 1985); en éste caso, el sujeto no observa relación entre su conducta y las consecuencias, percibiendo que su comportamiento depende de factores ajenos a su control.

Ya sabemos que las tres dimensiones referidas, en sus distintas subdimensiones, podrán mostrar un efecto diferencial a la hora de favorecer un comportamiento de aproximación e implicación en el deporte (López, 2000; González, Tabernero y Márquez, 2000; Torres, Carrasco y Medina, 2000). Va quedando claro, en los recientes trabajos de investigación, que también habrá de considerarse la forma en que se práctica la actividad física, así los jóvenes se acercan al deporte más por motivos intrínsecos que extrínsecos (Ryckman y Hamel, 1993; Villamarín, Mauri y Sanz, 1998; Torres, Carrasco y Medina, 2000); mientras que con el aumento en el nivel de competición se invierte esta influencia (González, Tabernero y Márquez, 2000).

Por otra parte, se han encontrado datos que nos indican que el género marca diferencias en los motivos y en la valoración que se realiza para la práctica deportiva. Las mujeres destacan las relaciones personales generadas en la práctica de la actividad deportiva como más importantes que para los chicos, señalándose el factor socializador del deporte como básico en la mujer; así como factores situacionales de cercanía a su domicilio (Torres, Carrasco y Medina, 2000). En la ciudad de León, González, Tabernero y Márquez (2000), analizando los motivos alegados para la práctica en deportes como el tenis; encuentran que los chicos dan más importancia que las chicas al "gusto por las emociones", por los "desafíos", "alcanzar un status", "hacer aquello en lo que destacan", "gastar energía" y "satisfacer a padres y amigos".

Así pues, podemos señalar que conocer el tipo de motivación manifestada por un sujeto para la práctica deportiva resulta básico y prioritario; y esto se ha visto reflejado, como ya apuntamos anteriormente, en el interés despertado entre los investigadores desde los años sesenta (Balaguer y Atienza, 1994). La finalidad que se persigue con dicho conocimiento sería permitirnos diseñar estrategias diferenciales de promoción, captación y mantenimiento en la ejecución de la actividad física, a través de entrenamientos específicos, ajustadas a cada una de las necesidades reconocidas por los participantes.

En años recientes hemos podido presenciar, cómo se ha atribuido a la presión social ejercida para alcanzar determinados cánones de belleza, el espectacular incremento de usuarios a los gimnasios $y$ consecuentemente la proliferación de éstos a lo largo de toda la geografía en nuestro país; generando con ello una actividad económica, social etc. de magnitudes relevantes. El 
fisicoculturismo, entendido como el entrenamiento con pesas para la musculación en su sentido amplio, ha venido marcado por una aureola de rechazo social por muy diversos motivos, llevando a realizar ajustes en la misma denominación como forma de atraer a nuevos practicantes sin que éstos puedan sentir el rechazo o estigma social (Arbinaga, Caracuel, en prensa). A pesar de la gran cantidad de personas que diariamente acuden a los gimnasios (¿se la podría considerar como la actividad física con mayor número de practicantes, superando incluso a deportes con mayor renombre?), con la finalidad de buscar un cuerpo musculoso, flexible y vascularizado; son pocos, y faltos de rigor, los datos aportados sobre los motivos que los usuarios manifiestan para invertir tanto tiempo, esfuerzo y dinero en acudir a estas salas de entrenamiento. En este sentido, se ha tendido a considerar principalmente, y casi en exclusiva, los motivos estéticos como aquellos que llevan a los culturistas a dedicar diariamente un número importante de horas entre pesas, mancuernas, barras y espejos; sin una mayor consideración en la determinación de otros factores motivacionales que pudieran observarse.

En este contexto, que brevemente hemos apuntado, el objetivo que pretendemos con este trabajo sería aportar los datos preliminares que nos van a permitir conocer el tipo de motivación alegada por los usuarios de los gimnasios y relacionarlo específicamente con variables del entrenamiento centradas en las pesas y otras actividades de musculación que realizan en el mismo.

\section{METODOLOGIA}

\section{Sujetos}

El total de sujetos participantes fue de 55 personas (40 varones y 15 mujeres) reclutados de diversos gimnasios de la ciudad de Huelva (España). La participación era voluntaria y solo se requería llevar más de seis meses entrenando de forma regular, al menos tres veces en semana. Elegimos este punto de corte en la selección al considerar que era tiempo suficiente como para que se eliminasen de la misma aquellos sujetos que no muestran una adherencia mínima al entrenamiento.

A los gimnasios se acudían cuatro días a la semana, alternando las mañanas y las tardes, el encuestador preguntaba a todo aquel que fuera entrando al gimnasio con la intención de entrenar, una vez que el preparador confirmase que cumplían el criterio establecido, si quería participar en cumplimentar un trabajo sobre el entrenamiento que realizaba. La prueba se rellenaba antes de entrenar.

\section{Instrumentos}

Para la recogida de información se confeccionó una hoja de respuestas organizada en tres bloques temáticos.

El primero de ellos contenía los datos de identificación básica, que se completaron con las siguientes variables: sexo, edad, peso y altura, con los que se calculaba el Índice de Masa Corporal (I.M.C), también conocido como Índice de Quételet (Garrow y Webster, 1985), satisfacción con la constitución física, valoración del peso y cumplimiento de dietas alimentarias.

En el segundo bloque temático se recogía información sobre la actividad deportiva: tiempo practicando el entrenamiento con pesas en gimnasios, motivo para el inicio a entrenar, número de días y horas entrenadas, echar de menos cuando no se entrena, intención de competir en los próximos años, comparaciones con otros deportistas en las salas de gimnasio y malestar producido.

El tercer apartado se compuso de " $\mathrm{La}$ Escala de Motivación en el Deporte" (E.M.D) traducida al castellano, y adaptada para la población de México, por López (2000), donde puede consultarse los valores psicométricos relevantes, y originariamente diseñada por Bière, Vallerand, Blais, y Pelletier, (1995). La E.M.D. presenta en su versión castellana siete factores, cuyas denominaciones aún provisionales (según el autor de la adaptación), varían con respecto a los nombres de la versión original que nosotros señalamos a continuación entre paréntesis (donde no se indica es que mantiene el mismo nombre): F.I.Amotivación, F.II.- Motivación Extrínseca 
(Regulación Externa), F.III.- Motivación Intrínseca Estética (Regulación Introyectada), F.IV.- Motivación Intrínseca- Autosuperada (Regulación Identificada), F.V.- Motivación Intrínseca al Conocimiento, F.VI.- Motivación Intrínseca al Cumplimiento y F.VII.Motivación Integral Deportiva (Motivación Intrínseca Estimulación). Por tanto, se observan como Motivación Externa a los factores: F.II, F.III, F.IV, como Motivación Interna se señalan a los factores: F.V, F.VI, F.VII, y por último la Amotivación estaría reflejada por el factor: F.I.

Las respuestas, a los 29 reactivos, se presentan en formato tipo Likert variando desde 1.- Nunca hasta 7 Siempre; eligiendo las que más se acerque a las razones por las que practica el entrenamiento deportivo. Si bien existe la posibilidad de transformación de los puntajes crudos en percentiles nosotros hemos optado por el trabajo con la puntuación directa en cada caso.

\section{RESULTADOS}

La muestra estaba integrada de 55 personas; y como era de esperar, la mayoría se compuso de hombres, en un $72.7 \%$, frente a las mujeres que sólo representaban un $27.3 \%$ del grupo. La edad media de la muestra fue de $26.65 \pm 10.53$ años. Los hombres son algo más jóvenes (25.45 \pm 10.53 años) que las mujeres (29.87 \pm 11.61 años), aunque no de forma significativa. En la Tabla 1 se deja constancia de las principales características sociales de la muestra y su relación con la variable sexo.

Cuando a los sujetos se les pregunta el motivo por el cual comenzaron a entrenar con pesas en el gimnasio podemos observar que el $41.8 \%$ dice haberlo hecho por mantener la forma física, el $29.1 \%$ para bajar peso, el $12.7 \%$ para competir, el $3.6 \%$ como forma de pasar el rato, el $1.8 \%$ por las sensaciones que les produce la actividad física, igual porcentaje por que lo hacían mis amigos y el $9.1 \%$ por otras razones.
Tabla 1. Características sociodemográficas de la muestra.

\begin{tabular}{lccc}
\hline & $\begin{array}{c}\text { \% } \\
\text { Total }\end{array}$ & $\begin{array}{c}\text { Hombres } \\
(\mathbf{n = 4 0})\end{array}$ & $\begin{array}{c}\text { Mujeres } \\
(\mathbf{n = 1 5})\end{array}$ \\
\cline { 2 - 4 } Sexo & & & \\
$\quad$ Hombre & 72.73 & & \\
$\quad$ Mujer & 27.27 & & \\
Edad & & & \\
$\quad$ De 14 a 19 & 21.8 & 25.0 & 13.3 \\
De 20 a 24 & 41.8 & 45.0 & 33.3 \\
De 25 a 31 & 9.1 & 10.0 & 6.7 \\
De 32 a 39 & 16.4 & 7.5 & 40.0 \\
De 40-60 & 10.9 & 12.5 & 6.7 \\
Estado Civil & & & \\
Soltero & 69.1 & 67.5 & 73.3 \\
Casado & 20.0 & 22.5 & 13.3 \\
Separ/Divorc. & 1.8 & 2.5 & ----- \\
Viudo & 1.8 & ----- & 6.7 \\
Otros. & 7.3 & 7.5 & 6.7 \\
Nivel de Estudios & & & \\
Sin Estudios & 9.1 & 10.0 & 6.7 \\
G.Escolar & 29.1 & 37.5 & 6.7 \\
Bachiller & 14.5 & 10.0 & 26.7 \\
F.Profesional & 23.6 & 22.5 & 26.7 \\
Univ. Medio & 14.5 & 10.0 & 26.7 \\
Univ. Superior & 9.1 & 10.0 & 6.7 \\
\hline
\end{tabular}

Si se analizan los motivos de inicio en función del sexo de los sujetos, veríamos que las mujeres reconocen en el $66.7 \%$ de los casos acudir al gimnasio para bajar peso, el $20 \%$ por la forma física y el $13.3 \%$ por otros motivos. Sin embargo, en el caso de los hombres, los motivos se muestran más variados; así, el 50\% lo hace por mantener la forma física, el $17.5 \%$ para competir, el $15 \%$ para bajar el peso, el $5 \%$ como forma de pasar el rato, con el $2.5 \%$ se observan los motivos de los amigos y las sensaciones derivadas del ejercicio físico y un $7.5 \%$ de los sujetos reconocen otros motivos. En la Tabla 2 reflejamos las características más genéricas del entrenamiento.

Podemos indicar que no se observan diferencias entre los sexos en el número de días que realizan entrenamientos, siendo la medía del grupo $4.5 \pm 0.8$ días a la semana (IC 95\% 4.3-4.7). De igual forma, tampoco se muestra diferencias, entre hombres y mujeres, en el número de horas que le dedican a la sesión de entrenamiento; en este sentido, se refleja que el $74.5 \%$ de los casos dice entrenar 
en sesiones que se varían entre 1 y 2 horas, el $14.5 \%$ le dedica más de 2 horas y el $10.9 \%$ dice hacerlo menos de 1 hora.

Tabla 2. Características de entrenamiento de la muestra.

\begin{tabular}{lccc}
\hline & Total & $\begin{array}{c}\text { Hombres } \\
(\mathbf{n = 4 0 )}\end{array}$ & $\begin{array}{c}\text { Mujeres } \\
(\mathbf{n = 1 5})\end{array}$ \\
\cline { 2 - 4 } & & & \\
I.M.C & & & \\
M \pm DS & 25.84 .2 & 25.23 .6 & 27.55 .2 \\
$\quad$ (IC-95\%) & $(24.7-26.9)$ & $(24.1-26.4)$ & $(24.6-30.3)$ \\
Tiempo & & & \\
$\begin{array}{l}\text { Entrenando \% } \\
\text { < de 1 año }\end{array}$ & & & \\
de 1 a 2 años & 18.5 & 47.5 & 40.0 \\
de 2 a 4 años & 18.2 & 17.5 & 20.0 \\
> de 4 años & 18.2 & 15.0 & 26.7 \\
\hline
\end{tabular}

Los resultados obtenidos por el conjunto de los sujetos, y en función de cada sexo, para cada uno de los siete factores que componen la escala E.M.D. se presentan, junto a los valores normativos de la adaptación en castellano, en la Tabla 3.

Tabla 3. Factores de motivación en la muestra (M $\pm \mathrm{DS}$ ) y valores normativos (López, 2000).

\begin{tabular}{lcccc}
\hline & $\begin{array}{c}\text { Total } \\
(\mathbf{n}=\mathbf{5 5})\end{array}$ & $\begin{array}{c}\text { Hombres } \\
(\mathbf{n}=\mathbf{4 0})\end{array}$ & $\begin{array}{c}\text { Mujeres } \\
(\mathbf{n}=\mathbf{1 5})\end{array}$ & $\begin{array}{c}\text { Valor } \\
\text { Normativo } \\
(\mathbf{n = 1 0 0 3})\end{array}$ \\
\cline { 2 - 5 } FI & 30.20 & 29.73 & 31.47 & 30.92 \\
& \pm 5.2 & \pm 5.6 & \pm 3.9 & \pm 4.5 \\
FII & 18.49 & 20.83 & 12.27 & 25.55 \\
& \pm 9.1 & \pm 8.5 & $\pm 8.0 *$ & $\pm 8.8 * *$ \\
FIII & 21.96 & 21.00 & 24.53 & 21.49 \\
& \pm 4.9 & \pm 4.5 & $\pm 5.5 * *$ & \pm 4.9 \\
FIV & 18.51 & 18.18 & 19.40 & 23.72 \\
& \pm 5.8 & \pm 5.6 & \pm 6.3 & $\pm 3.7 * *$ \\
FV & 16.56 & 17.70 & 13.53 & 21.22 \\
& \pm 6.9 & \pm 5.8 & \pm 8.9 & $\pm 4.9 * *$ \\
FVI & 20.78 & 20.58 & 21.33 & 24.66 \\
& \pm 5.7 & \pm 5.4 & \pm 6.6 & $\pm 3.1 * *$ \\
FVII & 9.16 & 9.45 & 8.40 & 11.66 \\
& \pm 3.4 & \pm 3.4 & \pm 3.3 & $\pm 2.2^{* *}$ \\
\hline$* p<0.001, * * p<0.05$ & &
\end{tabular}

Observamos que las mujeres puntúan de forma superior a los hombres en el factor FIII $(p<0.05)$ y éstos lo hacen en el factor FII $(p<0.001)$. Nuestro grupo sólo mantiene valores semejantes a los deportistas de la muestra utilizada para adaptar el instrumento en los factores FI (Amotivación) y FIII
(Motivación Intrínseca Estética o de Regulación Introyectada).

Por lo que se refiere a variables relacionadas con la práctica deportiva, podemos observar que la mayoría echa de menos entrenar los días que no lo hacen y reconocen ganas de ello en el $70.9 \%$ de los casos (Tabla 4).

A la pregunta de si cuando ven a un compañero de gimnasio más musculoso que él/ella, suelen pensar que les gustaría estar así; podemos comprobar, mediante el análisis de $\mathrm{Chi}^{2}$ Pearson, que hay una tendencia significativa a que las mujeres no suelan pensar en eso frente a los hombres que sí suelen pensarlo alguna vez $\left(\chi^{2}=11.10389, p=\right.$ 0.01118). De igual forma, aquellos que no suelen compararse, o no suelen pensar en ser tan musculoso como el compañero, muestran una tendencia a que no les gustaría competir, frente a los sujetos que siempre lo piensan cuando ven alguien mas voluminoso que sí les gustaría llegar a participar en una competición de fitness o culturismo $\left(\chi^{2}=9.98743, p=\right.$ 0.01867).

Por lo demás, no se observan diferencias, entre hombres y mujeres, en el deseo de competir, ni en las ganas de entrenar cuando no se acude al gimnasio; tampoco se dan en las dietas alimenticias, en la satisfacción con el físico y la valoración del peso. De igual forma, se observa una ausencia de diferencias entre los niveles de la variable comparación o el deseo de ser como el compañero más voluminoso y sus relaciones con la satisfacción con el físico, la valoración del peso y la práctica de dieta (Tabla 5).

Ahora bien, si analizamos los factores motivacionales evaluados, comparando el valor medio obtenido por aquellos sujetos que les gustaría competir y aquellos a los que no, nos encontramos que los primeros puntúan más alto de forma significativa en el factor FII $(p=0.011)$ y el factor FV $(p=0.005)$. De igual forma, aquellos sujetos que desean entrenar, o lo echan de menos, los días que no acuden al gimnasio, puntúan por encima de los sujetos a los que no les ocurre esto, en los factores FIII $(p=0.000)$ y en el factor FVI $(p=0.004)$.

Por su parte, si agrupamos a los sujetos en sólo dos niveles, no pensarlo nunca $\mathrm{y}$ 
pensarlo al menos alguna vez, ante la posibilidad de compararse o desear el volumen de los compañeros, vemos que estos últimos obtienen mayores puntuaciones en el factor FII $(p=0.013)$. En este mismo factor se muestran diferentes los sujetos a los que les gusta su cuerpo tal y como es, frente a los que o no les gusta nada o hay algunas parte de su cuerpo que no les gustan, quienes puntúan menos $(p=$ 0.026 ).

Tabla 4. Variables (\%) relativas a la práctica deportiva en función del sexo.

\begin{tabular}{lccc}
\hline & $\begin{array}{c}\text { Total } \\
(\mathbf{n = 5 5})\end{array}$ & $\begin{array}{c}\text { Hombres } \\
(\mathbf{n = 4 0})\end{array}$ & $\begin{array}{c}\text { Mujeres } \\
(\mathbf{n = 1 5})\end{array}$ \\
\cline { 2 - 4 } $\begin{array}{l}\text { Ganas de } \\
\text { entrenar }\end{array}$ & & & \\
$\quad$ Sí & 70.9 & 65.0 & 86.7 \\
$\quad$ No & 29.1 & 35.0 & 13.3 \\
$\begin{array}{l}\text { Deseo de } \\
\text { Competir }\end{array}$ & & & \\
$\quad$ Sí & 29.1 & 35.0 & 13.3 \\
$\quad$ NO & 70.9 & 65.0 & 86.7 \\
Comparaciones & & & \\
$\quad$ No & 45.5 & 32.5 & 80.0 \\
$\quad \begin{array}{l}\text { Sí. alguna vez } \\
\text { Sí. Muchas }\end{array}$ & 36.4 & 45.0 & 13.3 \\
$\quad$ veces & 5.5 & 5.0 & 6.7 \\
$\quad \begin{array}{l}\text { Sí. Siempre lo } \\
\text { pienso }\end{array}$ & 12.7 & 17.5 & ------ \\
\hline
\end{tabular}

\section{DISCUSIÓN}

Como hemos dicho al iniciar este trabajo, desde el ámbito de la ciencia se le ha dedicado por atención a la caracterización de los sujetos que realizan una actividad física como es la musculación o el entrenamiento con pesas en gimnasios. Y es debido a esta falta de datos que resulta difícil encontrar información con cierto rigor que nos permita contextualizar nuestros resultados.

Como era de esperar los sujetos que practican este tipo de entrenamientos son en su mayoría varones y jóvenes, detectándose un importante grupo de mujeres en la franja de edad 32 a 39 años. En su mayoría se han iniciado en la musculación mediante el levantamiento de pesas como forma de mantener la forma física, aunque en las mujeres el motivo dominante es el control del peso.
Nuestra muestra refleja que sólo mantiene valores similares, a los obtenidos por López (2000), en factores como la amotivación y como la motivación extrínseca de regulación introyectada (motivación intrínseca estética en terminología de éste autor); el resto de los factores muestran un menor peso que en el grupo utilizado para la adaptación de la escala. Las mujeres suelen indicar una mayor influencia de la regulación introyectada y un menor peso de la regulación externa, la cual se observa en los hombres con valores superiores. Esto podría relacionarse con la forma e intención en que se practica la actividad física, ya que son los hombres los que más desearían llegar a competir; y es a estos niveles, competición vs iniciación, donde la motivación extrínseca toma relevancia como se ha visto en otros lugares (Ryckman y Hamel, 1993, Tomas, Carrasco y Medina, 2000; González, Tabernero y Márquez, 2000).

Por otra parte, hemos intentado analizar algunas variables relevantes para la aparición de algunos trastornos asociados a esta práctica deportiva (Arbinaga y Caracuel, en prensa; Caracuel y Arbinaga, 2002); como es el caso de aquella que considera si al sujeto le gustaría ser igual de musculoso que otros compañeros de gimnasio cuando se compara con ellos y nos encontramos que ese deseo se hace mas fuerte en los varones frente a las mujeres, que no tienden a pensarlo. De igual forma aquellos que tienden a compararse reconocen que sí les gustaría competir en campeonatos de fitness o culturismo.

En este sentido, los sujetos que reconocen algún nivel de respuesta positiva en la pregunta de si les gustaría ser como los compañeros más voluminosos suelen diferenciarse de aquellos que nunca lo piensas en factores extrínsecos de regulación externa. Así mismo, los sujetos a los que les gustaría competir tienden a puntuar más en éste mismo factor, y además en la motivación intrínseca, en su dimensión referida al conocimiento, en acuerdo con otros trabajos (González, Tabernero y Márquez, 2000). Por su parte, los sujetos que se encuentran satisfechos con su constitución física, tal y como es, se ven principalmente influenciados por factores de regulación externa en mayor medida que 
aquellos quienes consideran que o no les gusta o piensan que alguna parte de su cuerpo no les satisface.

Vemos pues que los varones se rigen más por contingencias externas y muestran una mayor tendencia a compararse y a desear competir; señalándose en este último caso una mayor influencia dada por el deseo de conocer nuevas alternativas. Por último, se muestra también que aquellos sujetos que suelen observar, compararse y desear ser como aquellos compañeros mucho más voluminosos son a los que más les gustaría competir.

Tabla 5. Valoración (\%) de los aspectos físicos en función del sexo.

\begin{tabular}{lccc}
\hline & $\begin{array}{c}\text { Total } \\
\text { (n= 55) }\end{array}$ & $\begin{array}{c}\text { Hombres } \\
(\mathbf{n = 4 0 )}\end{array}$ & $\begin{array}{c}\text { Mujeres } \\
(\mathbf{n = 1 5})\end{array}$ \\
\cline { 2 - 4 } Satisfacción constitución Física. & & & \\
& 14.5 & 12.5 & 20.0 \\
No. para nada satisfecho & 60.0 & 57.5 & 66.7 \\
Sí. pero hay partes que no & 25.5 & 30.0 & 13.3 \\
$\quad$ Sí. me gusta mi cuerpo & & & \\
Valoración del Peso. & 3.6 & 2.5 & 6.7 \\
$\quad$ Muy por debajo de lo adecuado & 16.4 & 22.5 & ----- \\
Un poco por debajo de lo adecuado & 29.1 & 32.5 & 20.0 \\
Es adecuado & 40.0 & 35.0 & 53.3 \\
Un poco por encima del adecuado & 10.9 & 7.5 & 20.0 \\
Muy por encima del adecuado & & & \\
& & & \\
Cumplimiento de Dieta & 27.3 & 32.5 & 13.3 \\
No hago dieta & 38.2 & 30.0 & 60.0 \\
No hago. pero controlo los alimentos & 10.9 & 15.0 &.---- \\
Sí. pero no estricta & 23.6 & 22.5 & 26.7 \\
Sí. estricta y la respeto & & & \\
\hline
\end{tabular}

\section{CONCLUSIÓN}

A modo de conclusión podemos decir que el tópico del entrenamiento por factores meramente estéticos no parece mantenerse, o al menos no con la intensidad que muchas veces se le había atribuido.

Era de esperar la dominancia en el número de hombres. Pero son escasas las diferencias entre los sexos en el resto de variables; habiéndose encontrado diferencias sólo en la regulación externa, donde los hombres muestran un comportamiento más regido a través de medios externos, tales como premios y castigos; mientras la mujer refleja que ha producido una internalización de las razones para sus acciones o de pasadas contingencias externas.

Para un futuro próximo, vemos como interesante incrementar la atención que desde la psicología de la actividad física y del deporte se le dedica al fisicoculturismo, la musculación y el entrenamiento con pesas como única actividad deportiva realizada por los sujetos. Esto debe ser así, dado el volumen muy importante de practicantes que diariamente se implican en una actividad física que en muchos casos no cuenta con el apoyo de la "mass-media", llegándose incluso al rechazo social, al menos en nuestro país.

Como decimos, hay que realizar, desde la psicología, dicha apuesta en esta actividad física ya que en ella podemos ver reflejado cualquier proceso psicológico que pudiera aparecer en otros deportes. Desde los procesos motivacionales, de concentración, visualizaciones, cogniciones, hasta todos los aspectos relativos a la psicología del entrenamiento, actuaciones en la recuperación de lesiones, intervención y preparación a la competición y un largo etc de forma más genérica. A todo ello, habría que añadir la 
importancia que en este campo se le dedica a la alimentación como forma de controlar la composición corporal, y en muchos casos con los riesgos derivados de ello. Y creemos habría que prestar mayor atención no sólo desde la óptica de los trastornos asociados (vigorexia, anorexia, abuso de esteroides-anabolizantes etc...) sino desde la vertiente de la psicología de la normalidad y de los procesos básicos; como forma de mejorar el rendimiento de estos atletas.

\section{BIBLIOGRAFÍA}

Arbinaga, F y Caracuel, J.C (en prensa): Aproximación a la Dismorfia Muscular. Cuadernos de Medicina Psicosomática y Psiquiatría de Enlace.

Balaguer, I y Atienza, F (1994). Principales motivos de los jóvenes para jugar al tenis. Apunts, 31, 285-299.

Bière, N.M; Vallerand, RJ; Blais, MR y Pelletier, LG (1995). Dévelopement et validation d'une mesure de motivation intrinsèque, extrrinsèque et damotivation en contexte sportif: Léchelle de Motivation dans les Sports (ÉMS). International Journal of Sport Psychology, 26, 645-489.

Deci, E.L. (1971). Effects of externally mediated rewards on intrinsic motivation. Journal of Personality and Social Psychology, 18, 105-115.

Deci, EL y Ryan, RM (1985). Intrinsic Motivation and self determination in human behavior. N.Y: Plenum Press.

García Ferrando, M (1991). Los españoles y el deporte (1980-1990). Un análisis sociológico. Consejo Superior de Deportes. Madrid.

González, G; Tabernero, B y Márquez, S. (2000). Análisis de los motivos para participar en Fútbol y en Tenis en la iniciación deportiva. Revista Motricidad, 6, 47-66.
Gordillo, A (1992). Orientaciones psicológicas en la iniciación deportiva. Revista de Psicología del Deporte. 1, 27-36.

Gould, D (1982). Sport psychology in the 1980s: Status, direction, and challenge in younth sport research. Journal of Sport Psychology, 4, 203-218.

López, JM. (2000). Estandarización de la Escala de Motivación en el Deporte (EMD) de Brière NM, Vallerand, RJ; Blais, MR y Pelletier, LG. En deportistas mexicanos. Revista Motricidad, 6, 6793.

Morilla, M (1994). El papel del entrenador en la motivación deportiva. El Entrenador Español de Fútbol 60,26-31.

Ryckman, R y Hamel, J. (1993). Perceived physical ability differences in the sport participation motives of young athletes. International Journal of Sports Psychology, 24, 270-283.

Rodríguez, A. (1998). Estudio de las causas de abandono de la práctica deportiva habitual en la población de 14, 15 y 16 años. Tesis Doctoral. Universidad. Granada. España.

Torres, G; Carrasco, L y Medina, J (2000). Investigación sobre los motivos por los que los estudiantes universitarios practican deporte. El caso de la Universidad de Granada. Revista Motricidad, 6 , 95-105.

Vallerand, RJ y Halliwell, WR (1983). Formulations théoriques contemporaines en motivation intrinsèque. Revue et Critique. Psychologie Canadienne, 24, 243-256.

Villamarín, F; Mauri, C y Sanz, A. (1998). Competencia percibida y motivación durante la iniciación en la práctica del tenis. Revista de Psicología del Deporte, 13, 41-56. 